\title{
A Survey on Polarization Agile Antennas
}

\author{
M. Paranthaman
}

Assistant Professor, Department of ECE, M. Kumarasamy College of Engineering, Karur, India

\begin{abstract}
Antennas with dynamically choosing functionalities are the future demand for an efficient wireless communication system. Reconfigurable antennas are having such function to satisfy the need. It provides flexibility in frequency, pattern and diverse polarization. When compared to frequency and pattern reconfigurable antennas, polarization reconfigurable antenna provides better results while considering extenuating the losses due to multi channel transmission. Latest researches based on polarization diversity antennas are described in this paper to present the classifications.
\end{abstract}

Keyword: Circular patch; Polarization; Reconfigurable antennas; Switching.

\section{INTRODUCTION}

The demand for an efficient signal transmission in can be tuned between linear, left hand circular polarization wireless system increases with increasing number of and right hand circular polarization. Ring antenna with users[6]. Due to multipath transmission, mitigation of split able square used as slot here to switch the losses such as fading must be reduced to minimum level. polarization. Array with a size of $2 \times 2$ designed and Diversity in polarization must be achieved to extenuate the measured the important parameters. Special feature of this losses due to fading [3]. For this, antennas used for array is complementary nature of split square ring with transmitting and receiving should have the capability to TIY-5 substrate. Pin diodes are switching component change its polarization. Antennas which subject to change between the square slots.

its parameters, such as frequency, pattern and polarization called reconfigurable antennas. Frequency reusable antennas may offer wideband and different operations at time. Monopole antennas or loop antennas are the first to examine with multiple operations[12]. It was observed that complicated structure and high profile requiring huge quantity of diodes even though they offered expected results. Low profile antennas needed for easy design and fabrication to achieve better reconfigurability[8]. By using switches we can achieve polarization agility. Pin diodes[5], MEMS, varactor diodes, optical switches[2] and mechanically movable slots are the switching methods used in recent researches[9]. Some other switching types also proposed in various researchers and the detailed description will be discussed in later section of this paper.

The state of polarization can be changed from linear to circular or circular polarization itself it is subject change from left hand to right hand circular polarization[11]. Various researches proposed in polarization agile antennas. Two major design procedures followed in those investigations, they are arranging new feeding methods and changes in radiating area of an antenna.

\section{CLASSIFICATION}

The polarization agile antennas can be classified based on the switching methods used. Five types of polarization agile antennas explained in detail.

\subsection{Planar Polarization Agile Antenna}

Investigation based on substrate integrated waveguides gives planar polarization reconfigurability. Polarization

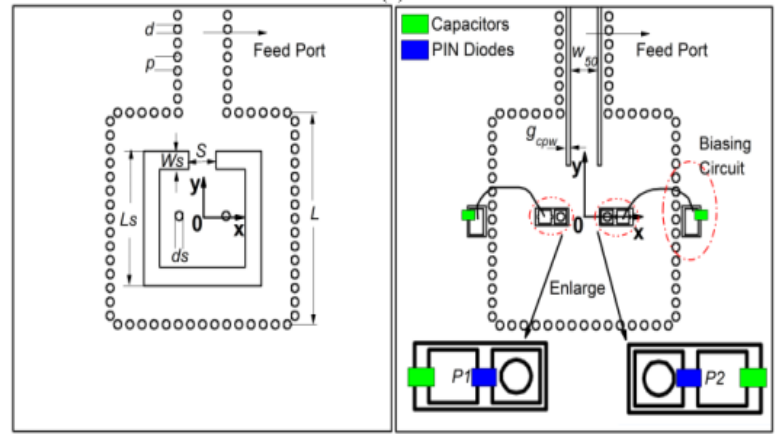

Figure 1 Planar Polarization agile antenna [1]

\subsection{C-Shaped Antenna with Polarization Reconfigurable}

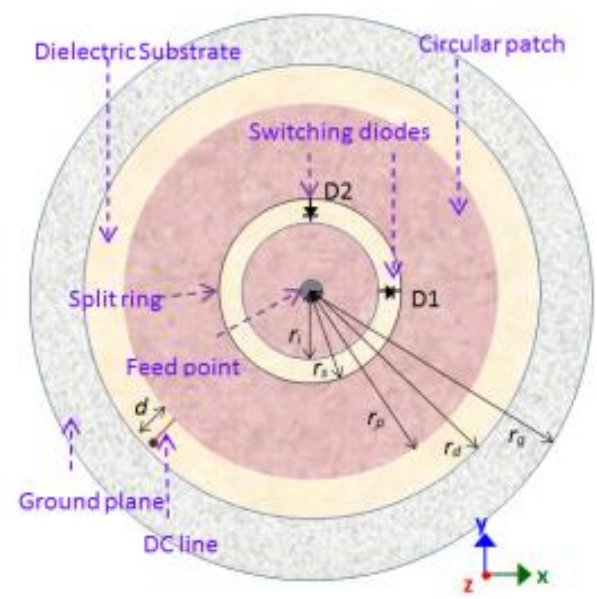

Figure 2 C-Shaped Polarization Reconfigurable antenna[4] 
Based on U-Slot structure, C-shaped antenna is designed The design of this antenna is carried out in multiple layers. for circular polarization. Center probe feeding is given to The layers are circular patch antenna, L-shaped this structure with a large change in concentric ring reconfigurable probe feeding and a bias line implemented antenna. As it is designed for polarization diversity, the as separate layer.

frequency of operation is fixed for the circular polarization. For switching diodes are used. It acts as a bridge for the inner and outer ring of the structure. If the switch is on state, the ring is split and for off state it is connected. Entire structure is placed on Rogers RT-Duroid substrate.

\subsection{Water Spiral Polarization Reconfigurable Antenna}

A novel tuning method to achieve polarization reconfigurability is proposed. For unidirectional radiation, the antenna put up at a ground plane. For left hand and right hand circular polarization, a glass container with two complementary channels is used. Spiral antenna with polarization diversity is achieved by directing the water flow into the channels. Despite of using conventional methods followed for spiral antenna, water is having high dielectric characteristics than metal strip which used in Cshaped antenna described above.

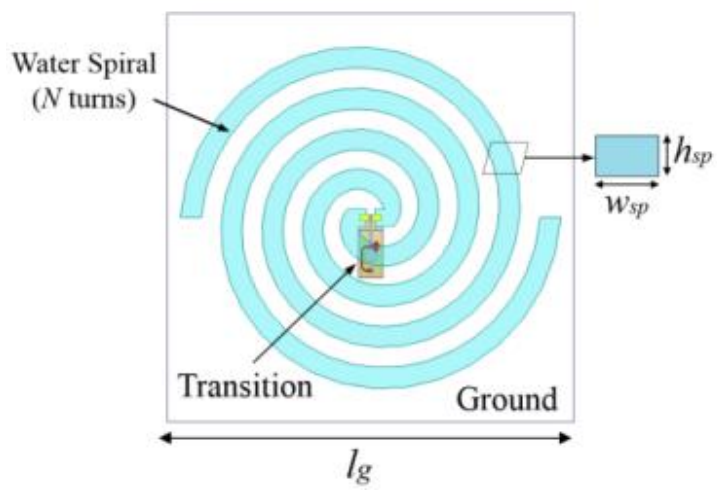

Figure 3 Water Spiral Polarization Antenna [7]

\subsection{L-Shaped Circular Patch Polarization Antenna}

Three different antennas with polarization reconfigurability explained above falls under the category of modifying the radiating structure. The L-shaped probe feeding to the Circular patch antenna offers multiple polarizations. Four states of operation can be achieved by using pin diodes for switching.

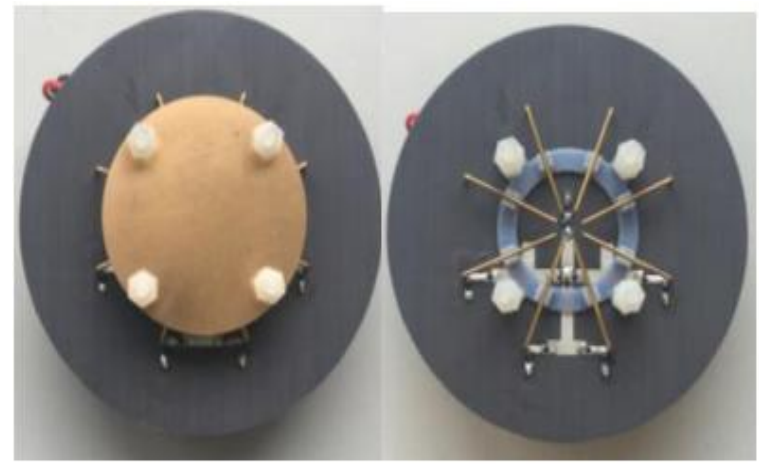

Figure 4 L-Shaped Reconfigurable Probe Feeding Antenna [10]

\subsection{Circular Polarized Reconfigurable Antenna}

Circular polarized antenna geometry is based on switching. The switching at radiating structure can be changed by using piezoelectric transducer. When the applying DC voltage gets changed the state of the antenna polarization will be changed from its current state. Here the antenna is designed for circularly polarized. For each change in dc voltage the polarization is changed from left hand circular polarization to right hand circular polarization and vice-versa.

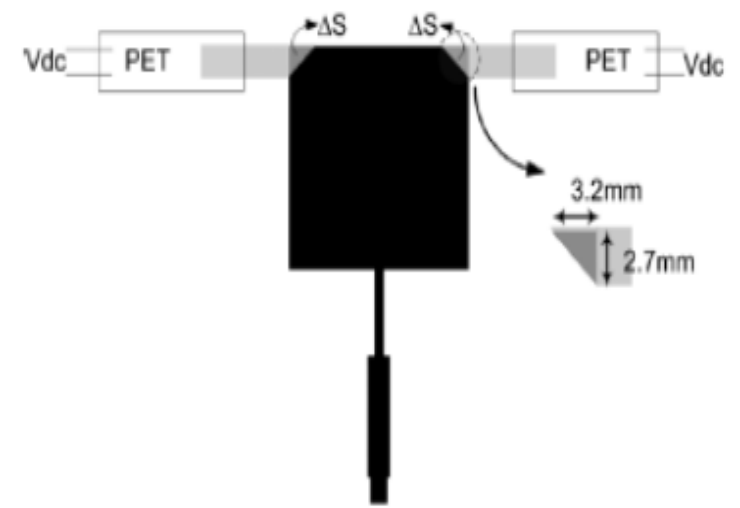

Figure 5 Circular Polarized Reconfigurable Antenna [13]

\section{CONCLUSION}

The classifications of polarization reconfigurable antennas are presented in this paper. Polarization agility is achieved through changing the radiating structure or by adapting to new feeding networks. Three types of polarization reconfigurability is obtained and they are linear polarization, left hand circular polarization and right hand circular polarization. To achieve the reconfigure nature diodes, transducers and even water can give switching between the slots. With these features polarization agile antennas are more suitable for wireless transmission.

\section{REFERENCES}

[1] Jun hu, Zhang-Cheng Hao, and Zhuo-Wei Miao (2016), " Design and Implementation of a Planar Polarization Reconfigurable Antenna" IEEE Antennas and Wireless Propagation Letters.

[2] V.Kavitha, V.Palanisamy(2013) "New Burst Assembly and Scheduling T technique for Optical Burst Switching Networks", Journal of Computer Science, Vol. 9, Issue 8, pp.1030-1040.

[3] S.Palanivel Rajan(2015) "Review and Investigations on Future Research Directions of Mobile Based Tele care System for Cardiac Surveillance", Journal of Applied Research and Technology, Vol.13, Issue 4, pp.454-460.

[4] Ka Ming Mak, Hau Wah Lai, Kwai Man Luk, and Kin Lun Ho(2016) "Polarization Reconfigurable Circular Patch Antenna with a C-Shaped"IEEE Transactions on Antennas and Propagation.

[5] S.Palanivel Rajan, M.Paranthaman, Dr.C.Vivek, (2016) "Design and Enhancement of Wideband Reconfigurability using Two EShaped Patch Antenna", Asian Journal of Research in Social 
Vol. 6, Issue 1, January 2017

Sciences and Humanities, ISSN : 2249-7315, Vol.6, Issue 9, pp. 317-327.

[6] C.Vivek, S.Palanivel Rajan(2016) "Z-TCAM : An Efficient Memory Architecture Based TCAM", Asian Journal of Information Technology, Vol.15, Issue 3, pp.448-454.

[7] Zhenxin Hu,Shiyan Wang,Zhongxiang Shen and Wen Wu(2016), "Broadband Polarization-Reconfigurable Water Spiral Antenna of Low Profile" IEEE Antennas and Wireless Propagation Letters.

[8] C.Vivek, S.Palanivel Rajan(2016) "Design of Data Aware Low Power Area Efficient Datapaths for Processing Elements in a Reconfigurable System", International Journal of Computer Science and Information Security, ISSN : 1947-5500, Vol.14, Issue 9, pp. 1100-1113.

[9] S.Palanivel Rajan(2014) "A Significant and Vital Glance on "Stress and Fitness Monitoring Embedded on a Modern Telematics Platform", Telemedicine and e-Health Journal, Vol.20, Issue 8, pp.757-758.

[10] S.Palanivel Rajan, M.Paranthaman, Dr.C.Vivek, (2016) "Design and Enhancement of Wideband Reconfigurability using Two EShaped Patch Antenna", Asian Journal of Research in Social Sciences and Humanities, ISSN : 2249-7315, Vol.6, Issue 9, pp. 317-327

[11] S.Palanivel Rajan, "Diagnosis of Cardiovascular Diseases using Retinal Images through Vessel Segmentation Graph", Current Medical Imaging Reviews, Online ISSN: 1875-6603, ISSN: 15734056, Vol. : 13, DOI : 10.2174/1573405613666170111153207, 2017..

[12] S.Palanivel Rajan, T.Dinesh(2015), "Systematic Review on Wearable Driver Vigilance System with Future Research Directions", International Journal of Applied Engineering Research, Vol. 2, Issue 2, pp.627-632.

[13] V.Kavitha, C.Gayathri, "A Survey on Detection Methods for Network Layer Attacks in WMN's", International Journal of Applied Engineering Research, Vol.10, Issue 1, pp.744-748, 2015.

[14] S.Palanivel Rajan, S.Vijayprasath(2015) "Performance Investigation of an Implicit Instrumentation Tool for Deadened Patients Using Common Eye Developments as a Paradigm", International Journal of Applied Engineering Research, Vol.10, Issue 1, pp.925-929.

[15] V.Kavitha, S.V.Vidya, "A grid based vehicle localization system providing safety precautions", International Journal of Applied Engineering Research, Vol.10, Issue 1, pp.940-944, 2015.

[16] Shih-Hsun Hsu, Kai Chang.(2007) "A Novel Reconfigurable Microstrip Antenna with Switchable Circular Polarization" IEEE Antennas and Wireless Propagation Letters, Vol. 6, pp.160-162.

[17] S.Palanivel Rajan, R.Sukanesh, "Experimental Studies on Intelligent, Wearable and Automated Wireless Mobile Tele-Alert System for Continuous Cardiac Surveillance", Journal of Applied Research and Technology, ISSN No.: 1665-6423, Vol. No. 11, Issue No.: 1, pp.133-143, 2013.

[18] V.Kavitha, V.Palanisamy, "Simultaneous Multi-path Transmission for Burst Loss Recovery in Optical Burst Switching Networks", European Journal of Scientific Research, Vol. 87, Issue 3, pp.412416, 2012.

[19] Agnus Swarnanisha Lakshmi, S.Palanivel Rajan, "Scheduled H and X Medicine Dispenser PoC Design", Journal of Chemical and Pharmaceutical Sciences, ISSN No.: 0974-2115, Special Issue : 8, pp. 1-4, 2016.

[20] V.Kavitha, C.Gayathri, "An Analysis on Routing and Issues in Network Layer in WMN's", International Journal of Scientific and Engineering Research, Vol. 6, Issue 4, pp.120-125, 2015.

[21] S. Palanivel Rajan, C.Vivek, "Blending Augmented Reality and Cloud - Need of the hour and an innovative approach", Journal of Chemical and Pharmaceutical Sciences, ISSN No.: 0974-2115, Special Issue : 8, pp. 23-27, 2016.

[22] C.Gayathri, V.Kavitha, "Mitigation of Colluding Selective Forwarding Attack in WMN's using FADE", International Journal for Trends in Engineering and Technology, Vol. 3, Issue 1, pp.6-12, 2015.

[23] S. Palanivel Rajan, M.Poovizhi, "Design of Patch Antenna Array for Radar Communication", Journal of Chemical and Pharmaceutical Sciences, ISSN No.: 0974-2115, Special Issue : 8, pp. 38-40, 2016.
[24] R.Dhivya, V.Kavitha, "Secured Client Cache Sustain for Maintaining Consistency in manet's", International Journal of Research in Engineering and Tech., Vol.3, Issue7, pp.1-6, 2014.

[25] C.Vivek, S. Palanivel Rajan, "Review of Low Power and High Speed Implementation of 3-bit Flash Analog to Digital Converter", Journal of Chemical and Pharmaceutical Sciences, ISSN No.: 09742115, Special Issue : 8, pp. 74-78, 2016.

[26] S.Palanivel Rajan, R.Sukanesh, "Viable Investigations and Real Time Recitation of Enhanced ECG Based Cardiac Tele-Monitoring System for Home-Care Applications: A Systematic Evaluation”, Telemedicine and e-Health Journal, ISSN: 1530-5627, Online ISSN: 1556-3669, Vol. No.: 19, Issue No.: 4, pp. 278-286, 2013.

[27] S.Palanivel Rajan, et.al., "Intelligent Wireless Mobile Patient Monitoring System", IEEE Digital Library Xplore, ISBN No. 9781-4244-7769-2, INSPEC Accession Number: 11745297, IEEE Catalog Number: CFP1044K-ART, pp. 540-543, 2010.

[28] S.Palanivel Rajan, et.al., "Cellular Phone based Biomedical System for Health Care", IEEE Digital Library Xplore, ISBN No. 978-14244-7769-2, INSPEC Accession Number: 11745436, IEEE Catalog Number: CFP1044K-ART, pp.550-553, 2010.

[29] S.Palanivel Rajan, et.al., "Performance Evaluation of Mobile Phone Radiation Minimization through Characteristic Impedance Measurement for Health-Care Applications", IEEE Digital Library Xplore, ISBN : 978-1-4673-2047-4, IEEE Catalog Number: CFP1221T-CDR, 2012.

[30] S.Palanivel Rajan, et.al., "Experimental Explorations on EOG Signal Processing for Real Time Applications in LabVIEW", IEEE Digital Library Xplore, ISBN : 978-1-4673-2047-4, IEEE Catalog Number: CFP1221T-CDR, 2012. 